\title{
Efeito do fósforo na captação de minerais e pigmentação de Azolla caroliniana Willd. (Azollaceae)
}

\author{
PAULO R. ADALBERTO ${ }^{1}$, ANTONIO C. MASSABNI ${ }^{1}$, ANTONIO J. GOULART ${ }^{2}$, \\ RUBENS MONTI ${ }^{2,4}$ e PEDRO M. LACAVA ${ }^{3}$
}

(recebido: 22 de agosto de 2003; aceito: 20 de maio de 2004)

\begin{abstract}
Effect of the phosphorus on the mineral uptake and pigmentation of Azolla caroliniana Willd. (Azollaceae)). Mineral bioaccumulation, $\mathrm{N}_{2}$ fixation and pigment accumulation of $A$. caroliniana were established under nutritional stress. Cultures were conduced in polystyrene flasks using phosphate gradient between $10^{-7}$ and $10^{-3} \mathrm{~mol}_{\mathrm{L}} \mathrm{L}^{-1}$. Minerals ( $\mathrm{P}, \mathrm{S}, \mathrm{B}$, and Sn) were quantified by induced coupled plasma and total nitrogen by Kjeldhal methodology. Nitrogenase activity was determined by the reaction of acetylene reduction and pigments were photometrically determined in acidified methanolic extracts. Macronutrients uptake (N, P, S) were shown to be related to phosphorus supplements and micronutrient absorption (B and Sn) was independent of phosphorous supplements. Mineral selectivity showed that A. caroliniana can act as biofertilizer. Phosphorus concentration below $10^{-5} \mathrm{~mol} . \mathrm{L}^{-1}$ decreased the ratio chlorophylls/anthocyanins causing reddening of the plant. This behaviour of $A$. caroliniana could be used for biomonitoring environmental phosphorus availability.
\end{abstract}

Key words - anthocyanins, Azolla caroliniana, mineral bioaccumulation, nitrogenase

RESUMO - (Efeito de fósforo na captação de minerais e pigmentação de Azolla caroliniana Willd. (Azollaceae)). O bioacúmulo de minerais, a fixação de nitrogênio e a concentração de pigmentos em Azolla caroliniana foram estabelecidos sob estresse nutritivo. As culturas foram mantidas em frascos de poliestireno, utilizando gradiente de fosfato entre $10^{-7}$ e $10^{-3}$ mol.L $\mathrm{L}^{-1}$. Os minerais (P, S, B e Sn) foram quantificados por espectrometria de emissão ótica com plasma de acoplamento induzido e o teor de nitrogênio pelo método de Kjeldahl. A atividade da nitrogenase foi determinada pela reação de redução do acetileno e as concentrações de pigmentos foram determinadas por fotometria nos extratos metanólicos acidificados. A absorção de macronutrientes (N, P, S) mostrou-se relacionada ao suprimento de fósforo, enquanto a absorção de micronutrientes (B e Sn) não sofreu este efeito. A seletividade mineral demonstra a potencialidade da A. caroliniana como biofertilizante. Para concentrações de fósforo abaixo de $10^{-5} \mathrm{~mol} . \mathrm{L}^{-1}$, a razão das clorofilas/antocianinas diminuiu, causando o avermelhamento do vegetal. Este comportamento de A. caroliniana poderia ser empregado para o biomonitoramento da disponibilidade de fósforo no ambiente.

Palavras-chave - antocianina, Azolla caroliniana, bioacumulação de minerais, nitrogenase

\section{Introdução}

A fixação biológica do nitrogênio contribui anualmente com cerca de $1,72 \times 10^{7}$ toneladas de nitrogênio reduzido para a produção vegetal em terras aráveis e em sistemas naturais (Ishizuka 1992). É grande o interesse nesta fonte de nitrogênio, tanto por razões econômicas, quanto pela busca de alternativas

\footnotetext{
1. Universidade Estadual Paulista "Júlio de Mesquita Filho" Instituto de Química, Departamento de Química Geral e Inorgânica, Rua Prof. Francisco Degni, s/n, Caixa Postal 355, 14801-970 Araraquara, SP, Brasil.

2. Universidade Estadual Paulista "Júlio de Mesquita Filho" Faculdade de Ciências Farmacêuticas, Departamento de Alimentos e Nutrição, Rod. Araraquara-Jaú, Km 1, Caixa Postal 502, 14801-902 Araraquara, SP, Brasil.

3. Universidade de Taubaté, Departamento de Ciências Agrárias, Estrada Municipal José Luiz Cembranelli, 5000, 12081-010 Taubaté, SP, Brasil.

4. Autor para correspondência: montiru@fcfar.unesp.br
}

menos agressivas ao ambiente (Kundu \& Ladha 1995). O pleno emprego de organismos fixadores de nitrogênio em culturas em tanques, contudo, encontra restrições que ainda os tornam pouco atrativo ao produtor, como altos custos, necessidade de grande área, de mão de obra qualificada e da utilização de grande quantidade de água (Ali 1999).

Azolla caroliniana, uma pteridófita aquática de pequeno porte, apresenta-se como um recurso verde de grande potencial para campos inundados. A simbiose com a cianobactéria diazotrófica Anabaena azollae contribui para o fornecimento de nitrogênio para seu crescimento. $\mathrm{O}$ feto tem ampla distribuição geográfica e se desenvolve bem em ambientes poluídos, quando comparado com outras espécies vegetais (Kondo et al. 1989, Singh \& Singh 1995). Quando exposta a altas concentrações de chumbo, níquel, cádmio, zinco, arsênio, ou outros metais pesados (Sela et al. 1990, Lales et al. 1993, Gaur et al. 1994), a planta produz peptídeos simples, as chamadas fitoquelatinas, as quais se 
complexam com os íons metálicos, promovendo a desintoxicação do vegetal (Paywall et al. 1993). Testes de toxicidade em Spirodela polyrhiza (L.) Schleid. e Azolla pinnata $\mathrm{R}$. Br. comprovaram a eficiência do vegetal como biofiltro contra uma série de metais pesados (Lales et al. 1993, Querubin et al. 1993, Gaur et al. 1994, Forni et al. 2001).

As limitações do cultivo de A. caroliniana são excesso de luminosidade, altas temperatura (Kondo et al. 1989, Singh \& Singh 1995) e fatores nutricionais (Singh \& Singh 1989, Cadiz \& Alejar 1992). Os efeitos da deficiência de fósforo são freqüentemente citados (Bisoyi \& Singh 1988, Bieleski \& Läuchli 1992, Kundu \& Ladha 1995). Nestas condições, ocorre uma falência generalizada do metabolismo da planta, acompanhada de sensível produção de pigmentos, o que causa o avermelhamento de suas frondes (Cadiz \& Alejar 1986, 1992).

Com o objetivo de estudar o uso de A. caroliniana como biofertilizante, procurou-se neste trabalho avaliar o acúmulo de macronutrientes (nitrogênio, fósforo e enxofre) e de micronutrientes (estanho e boro) em função da disponibilidade de fósforo, avaliar a produção de pigmentos e o desenvolvimento da planta ante a carência de fósforo solúvel e, ainda, determinar a atividade diazotrófica de $A$. caroliniana em diferentes concentrações de fósforo.

\section{Material e métodos}

Crescimento - a linhagem CENA-38 de Azolla caroliniana Willd. (Azollaceae) foi gentilmente fornecida pela Profa. Dra. Siu Mui Tsai do LBCM do Centro de Energia Nuclear na Agricultura/CENA - Piracicaba, SP. O cultivo foi conduzido em casa de vegetação, com variações de temperaturas mínimas diárias, registradas entre 20 e $23^{\circ} \mathrm{C}$ e máximas entre 29 e $35^{\circ} \mathrm{C}$. Utilizou-se frascos de poliestireno de $1 \mathrm{~L}$, nos quais, adicionou-se soluções nutritivas de Lumpkin, com concentrações de $\mathrm{Na}_{2} \mathrm{HPO}_{4}$ variando entre $10^{-7}$ mol. $\mathrm{L}^{-1} \mathrm{e}$ $10^{-3} \mathrm{~mol} \cdot \mathrm{L}^{-1}$. Estes limites correspondem, respectivamente, à um sério estresse para o crescimento e doses suficientes de fósforo solúvel (Kondo et al. 1989, Bieleski \& Läuchli 1992). Para definir a velocidade específica de crescimento em $\mathrm{mm}$, inóculos de aproximadamente $1 \mathrm{~g}$ de massa fresca foram cultivados por 30 dias. $\mathrm{O}$ aumento de biomassa foi registrado a cada 3 dias, quando, então, a cultura foi drenada e seca em discos de papel absorvente por alguns segundos e sua biomassa fresca foi determinada. A solução de cultura foi renovada e a evapotranspiração compensada com água desmineralizada, sempre que necessário (Lejeune et al. 2000). Os experimentos foram conduzidos em triplicatas $(n=3)$ e os resultados são apresentados como médias independentes, com respectivos desvios. O teste de Tuckey foi aplicado e os valores considerados semelhantes com $10 \%$ de significância $(\mathrm{p} \leq 0,10)$.

Atividade da nitrogenase - a atividade da nitrogenase foi determinada em triplicatas pelo ensaio da redução do acetileno. Frascos transparentes de $250 \mathrm{~mL}$, contendo $50 \mathrm{~mL}$ do meio de cultura e o vegetal íntegro, com biomassa determinada, foram lacrados com tampões de borracha. A troca gasosa foi realizada com a mistura nitrogênio/acetileno (9:1). O ensaio ocorreu em ambiente iluminado e climatizado a $25^{\circ} \mathrm{C}$. A cada duas horas, um volume de $0,5 \mathrm{~mL}$ da fase gasosa foi submetido a quantificação por cromatografia gasosa (Instrumentos Científicos CG Ltda., mod. 370, coluna Porapack $\mathrm{N}$ de $1,8 \mathrm{~m}, 3 / 16$ ") por um período de $24 \mathrm{~h}$. Brancos de reação foram preparados em frascos sem os vegetais. Os valores da atividade específica foram expressos em mmoles de $\mathrm{C}_{2} \mathrm{H}_{2} \cdot \mathrm{h}^{-1} \cdot \mathrm{g}^{-1}$ (Santos \& Lacava 1981).

Análise dos pigmentos vegetais - após a remoção das raízes, as frondes foram lavadas com água deionizada para remoção de qualquer resíduo do meio de cultivo. Após secagem em papel de filtro, foram submetidas a trituração em almofariz. Os pigmentos foram extraídos a partir de $0,1 \mathrm{~g}$ de amostra com $1,0 \mathrm{~mL}$ de metanol acidificado ( $\mathrm{HCl} 1 \%)$. Após a centrifugação a $9.000 \mathrm{rpm}$, por $15 \mathrm{~min}$, a $4{ }^{\circ} \mathrm{C}$, o sobrenadante foi coletado e seu volume ajustado para 5,0 $\mathrm{mL}$. Os pigmentos foram quantificados em extratos frescos num espectrofotômetro Ultrospec $1000 \mathrm{UV} / \mathrm{Vis}$ (Amersham Biosciences), em $\lambda 580 \mathrm{~nm}$ para antocianinas totais (Cadiz \& Alejar 1986) e $\lambda 660 \mathrm{~nm}$ para clorofila (MacFarlane \& Burchett 2001).

Análise de teores minerais - neste procedimento foi utilizada a planta íntegra lavada com água desmineralizada. Após secagem em papel de filtro e trituração, $0,1 \mathrm{~g}$ da amostra foi submetida à digestão ácida $\left(10 \mathrm{~mL}\right.$ de $\left.\mathrm{HNO}_{3}, 60^{\circ} \mathrm{C}, 6 \mathrm{~h}\right)$. Em seguida, a solução foi filtrada e diluída até atingir $100 \mathrm{~mL}$. As concentrações de P, S, B e Sn foram determinadas por espectrometria de plasma de acoplamento induzido. $\mathrm{Na}$ análise de $\mathrm{N}$ total foi empregada a metodologia de Kjeldhal (AOAC 1980).

\section{Resultados e Discussão}

Os resultados relativos ao efeito do fósforo no acúmulo de nutrientes de A. caroliniana são apresentados nas figuras 1 e 2 . Estes resultados permitem o reconhecimento de curvas que diferenciam macro e micronutrientes. Em quantidades suficientes de fósforo, o crescimento é acelerado e a demanda de macronutrientes é alta (tabela 1). Para o fósforo, concordando com os dados disponíveis na literatura (Kondo et al. 1989, Singh \& Singh 1989, Bieleski \& Läuchli 1992, Kundu \& Ladha 1995, Forni et al. 2001), foi observada absorção do nutriente consonante com sua concentração no meio (figura 1). O metabolismo da fixação de nitrogênio da simbiose A. carolinianaAnabaena azollae, cultivada no campo ou sob 


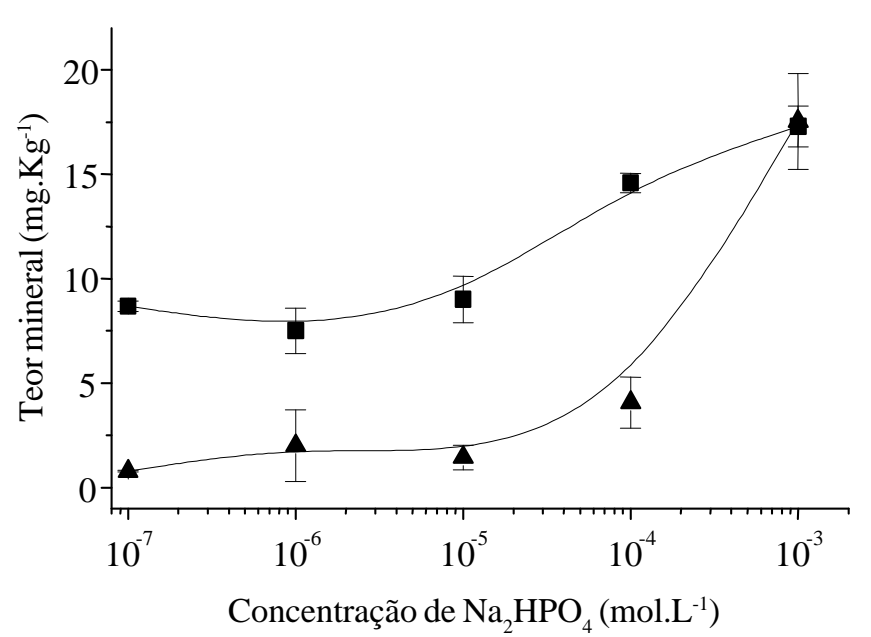

Figura 1. Influência da disponibilidade de fósforo no acúmulo de nitrogênio e fósforo em Azolla caroliniana. = = nitrogênio; - = fósforo. Os dados estão apresentados como médias \pm desvio padrão $(\mathrm{n}=3, \mathrm{p} \leq 10 \%)$.

Figure 1. Influence of the availability of phosphorus in the nitrogen and phosphorus accumulation in Azolla caroliniana $\mathbf{-}=$ nitrogen; $\boldsymbol{\Delta}=$ phosphorus. The data are presented as mean \pm standard deviation $(n=3, p \leq 10 \%)$.

condições controladas, declinou em estresse por deficiência de fósforo. Essa redução na atividade fixadora da cianobacteria, ilustrada na tabela 1, está refletida nos menores teores de nitrogênio acumulado nos tecidos obtidos de A. caroliniana sob tais condições. Com suprimento satisfatório de nutriente, os teores de nitrogênio tissular aumentaram em aproximadamente 17 vezes (figura 1).

A figura 2 mostra que a curva de assimilação de enxofre guarda forte semelhança com a de nitrogênio (figura 1), ainda que este seja requerido em menores quantidades. Nas concentrações experimentais, não foram atingidos níveis para se detectar inibição por fósforo, conforme anteriormente relatado por Bieleski \& Läuchli (1992). A análise das curvas indica que variações na concentração de nutriente da ordem de $10^{4}$ vezes não provocaram diferenças sensíveis na absorção de boro e estanho no material biológico em estudo (figura 2).

Pela tabela 1 é possível reconhecer o efeito negativo da deficiência de fósforo sobre a fixação de nitrogênio e na velocidade de crescimento do organismo. A redução de acetileno ficou comprometida, assim como a velocidade específica de crescimento, medida em $\mathrm{m}$ (constante de crescimento de Jacob e Monod). A partir dos resultados desta mesma tabela, foi possível determinar um valor de $\mathrm{K}_{\mathrm{s}}$ igual a $10^{-5} \mathrm{~mol} \cdot \mathrm{L}^{-1}$ de fósforo,

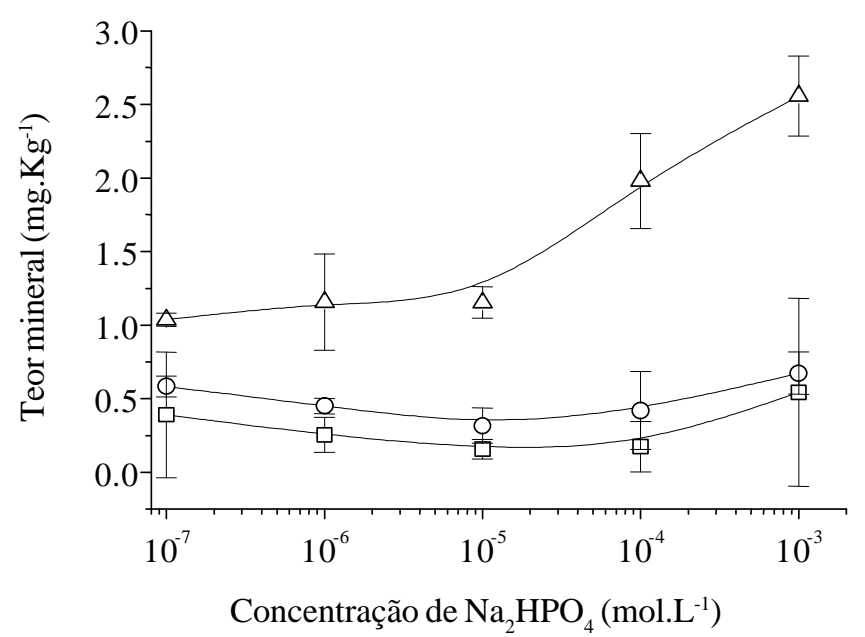

Figura 2. Influência da disponibilidade de fósforo no acúmulo de enxofre, boro e estanho em Azolla caroliniana. $\Delta=$ enxofre; $O=$ boro; $\square=$ estanho. Os dados estão apresentados como médias \pm desvio padrão $(\mathrm{n}=3, \mathrm{p} \leq 10 \%)$.

Figure 2. Influence of the phosphorus availability in the sulphur, boron and tin accumulation in Azolla caroliniana. $\triangle=$ sulphur; $O=$ boron; $\square=$ tin. The data are presented as mean \pm standard deviation $(\mathrm{n}=3, \mathrm{p} \leq 10 \%)$.

que é a concentração necessária para que a planta atinja metade da velocidade específica de crescimento.

A tabela 2 mostra a diferença dos pigmentos acumulados nas frondes do vegetal em culturas nas diferentes concentrações experimentais de fósforo. Esses resultados indicam que o teor de antocianinas aumenta quando os níveis de fósforo encontram-se

Tabela 1. Atividade da nitrogenase e a velocidade específica do crescimento de Azolla caroliniana em diferentes concentrações de fósforo. (Média \pm desvio padrão; $\mathrm{n}=3$, $\mathrm{p} \leq 10 \%$ ). Os controles de reação foram preparados em frascos sem os vegetais.

Table 1. Nitrogenase activity and growth specific velocity of Azolla caroliniana in different phosphorus concentrations. (Average \pm standard deviation; $\mathrm{n}=3, \mathrm{p} \leq 10 \%$ ). The blanks were prepared in flasks without the plants.

\begin{tabular}{ccc}
\hline $\begin{array}{c}\text { Concentração de } \\
\text { fósforo }\left(\mathrm{mol} . \mathrm{L}^{-1}\right)\end{array}$ & $\begin{array}{c}\text { Atividade da } \\
\text { nitrogenase } \\
\left(\mu \mathrm{mols} \mathrm{C}_{2} \mathrm{H}_{2} \cdot \mathrm{h}^{-1} \cdot \mathrm{g}^{-1}\right)\end{array}$ & $\begin{array}{c}\text { Velocidade específica } \\
\text { de crescimento } \\
\left(\mu \cdot \text { dia }^{-1}\right)\end{array}$ \\
\hline $10^{-3}$ & $7,28 \pm 0,15$ & $0,28 \pm 0,02$ \\
$10^{-4}$ & $3,78 \pm 0,31$ & $0,22 \pm 0,04$ \\
$10^{-5}$ & $2,44 \pm 0,29$ & $0,14 \pm 0,01$ \\
$10^{-6}$ & $1,67 \pm 0,22$ & $0,10 \pm 0,00$ \\
$10^{-7}$ & $1,96 \pm 0,36$ & $0,10 \pm 0,00$ \\
\hline
\end{tabular}


Tabela 2: Efeito de diferentes concentrações de fósforo sobre teores de clorofila e antocianina total e a coloração de Azolla caroliniana. (Média \pm desvio padrão; $\mathrm{n}=3, \mathrm{p} \leq 10 \%$ ).

Table 2. Effect of different phosphorus concentrations on the levels of total chlorophyll and anthocyanin and the coloration of Azolla caroliniana. (Average \pm standard deviation; $\mathrm{n}=3, \mathrm{p} \leq 10 \%$ ).

\begin{tabular}{ccccc}
\hline $\begin{array}{c}\text { Concentração de fósforo } \\
\text { no meio }\left(\mathrm{mol} . \mathrm{L}^{-1}\right)\end{array}$ & $\begin{array}{c}\text { Clorofila total } \\
\text { (Abs 660nm) }\end{array}$ & $\begin{array}{c}\text { Antocianina total } \\
(\text { Abs 580 nm) }\end{array}$ & $\begin{array}{c}\text { Abs 660 nm/ } \\
\text { Abs 580 nm }\end{array}$ & $\begin{array}{c}\text { Coloração da fronde } \\
10^{-3}\end{array}$ \\
$10^{-4}$ & $0,520 \pm 0,015$ & $0,123 \pm 0,025$ & 4,22 & verde \\
$10^{-5}$ & $0,210 \pm 0,025$ & $0,049 \pm 0,015$ & 1,32 & verde \\
$10^{-6}$ & $0,105 \pm 0,011$ & $0,075 \pm 0,011$ & 0,39 & vermelha \\
$10^{-7}$ & $0,065 \pm 0,008$ & $0,167 \pm 0,008$ & 0,21 & vermelha \\
\hline
\end{tabular}

abaixo de $10^{-5} \mathrm{~mol} \cdot \mathrm{L}^{-1}$. Nesta condição, a produção de antocianinas eleva-se até cinco vezes e, de modo inverso, as concentrações de clorofila são reduzidas à medida em que o fósforo disponível no meio torna-se escasso. As mudanças na pigmentação das folhas podem ser observadas a partir do ponto em que a relação de concentrações de clorofila/antocianina atinge valores abaixo de $1,4 \mathrm{e}$ as condições de crescimento tornam-se insatisfatórias.

A capacidade de acumular minerais atraiu o interesse para que A. caroliniana seja utilizada no tratamento de efluentes ricos em minerais, quer sejam urbanos, rurais ou naturais (Sela et al. 1990, Paywall et al. 1993, Uheda \& Nakamura 2000). Se, entretanto, esta característica tem tal aplicação, poderia, por outro lado, tornar o biofertilizante uma fonte de íons tóxicos no solo, devido à alta tolerância de vegetais, cujos ambientes se caracterizem pela concentração elevada destes íons (MacFarlane \& Burchett 2001).

$\mathrm{O}$ trabalho ora apresentado, indica que o manejo correto da A. caroliniana pode evitar a contaminação do biofertilizante, mantendo-se, com isso, os níveis de macro e micronutrientes requeridos pelo solo. Uma oportuna abordagem consiste na proposição de sistemas de produção de biomassa de A. caroliniana que façam uso de efluentes como substrato para o tratamento de resíduos e a produção de biofertilizantes. Os resultados obtidos apontam para uma concordância entre os valores dos dados nutricionais e as respostas do vegetal, o que permite fundamentar um método de análise quantitativa biomonitorada de fósforo ambiental. A razão de pigmentos e a disponibilidade ambiental de fósforo podem ser aplicadas como método de biosensoreamento na determinação deste nutriente no campo. Pode-se propor um "ponto de viragem" na pigmentação do vegetal para a concentração de fósforo em torno de $10^{-5} \mathrm{~mol}^{-L^{-1}}$ na solução de cultivo. Esta avaliação visual, embora indireta, possui a vantagem de ser pronta e facilmente aplicável.

Agradecimentos - $\mathrm{O}$ autor Paulo Roberto Adalberto agradece à Capes pela bolsa de estudos concedida.

\section{Referências bibliográficas}

ALI, M. 1999. Evaluation of green manure technology in tropical lowland rice systems. Field Crops Research 61:61-78.

AOAC. 1980. Officials methods of analysis. $13^{\text {th }}$ ed. ASSOCIATION OF OFFICIAL ANALYTICAL CHEMISTS, Washington.

BIELESKI, R.L. \& LÄUCHLI, A. 1992. Phosphate uptake, efflux and deficiency in the water fern, Azolla. Plant Cell and Environment 15:665-673.

BISOYI, R.N. \& SINGH, P.K. 1988. Effect of phosphorous fertilisation on blue green algal inoculun production and nitrogen yeld under field conditions. Biology and Fertility of Soils 4:338-343.

CADIZ, N.M. \& ALEJAR, A.A. 1986. Effects of abscisic acid and indole acid on nitrogen fixation and anthocyanin formation in Azolla pinnata (R. Brown). Philippine Agriculturist 69:89-101.

CADIZ, N.M. \& ALEJAR, A.A. 1992. The effects of abscisic acid and indole acid on nitrogen fixation and anthocyanin formation in Azolla pinnata. Reaserch in Photosynthesis 4:107-110.

FORNI, C., CHEN, J., TANCIONI, L. \& CAIOLA, M.G. 2001. Evaluation of the fern Azolla for growth, nitrogen and phosphorus removal from wastewater. Water Research 35:1592-1598.

GAUR, J.P., NORAHO, N. \& CHAUHAN, Y.S. 1994. Relationship between heavy metal accumulation and toxicity in Spirodela polyrhiza (L.) Schleid and Azolla pinnata R. Br. Aquatic Botany 49:183-192. 
ISHIZUKA, J. 1992. Trends in biological nitrogen-fixation research and application. Plant and Soil 141:197-209.

KONDO, M., KOBAYASHI, M. \& TAKAHASHI, E. 1989. Effect of phosphorous in Azolla and its utilization in rice culture in Niger. Plant and Soil 120:165-170.

KUNDU, D.K. \& LADHA, J.K. 1995. Enhancing soil-nitrogen use and biological nitrogen-fixation in wetland rice. Experimental Agriculture 31:261-277.

LALES, J.S., PAYWALL, P.C., QUERUBIN, L.J. \& PAGAPAS, V. 1993. Azolla as biological scavenger of geothermal ions III. Arsenic and boron uptake under field conditions. Philippine Agriculturist 76:185-191.

LEJEUNE, A.,PENG J., LEBOULENGE, E., LARONDELLE, Y. \& VAN HOVE, C. 2000. Carotene content of Azolla and its variations during drying and storage treatments. Animal Feed Science and Technology 5:295-301.

MACFARLANE, GR. \& BURCHETT, M.D. 2001. Photosynthetic pigments and peroxidase activity as indicators of heavy metal stress in the grey mangrove, Avicennia marina (Forsk.). Marine Pollution Bulletin 42:233-240.

PAYWALL, P.C., MACALE, M.A.R., LALES, J.S. \& QUERUBIN, L.J. 1993. Azolla as biological scavenger of geothermal ions I. Propagation and characterization of selected Azolla variants. Philippine Agriculturist 76:73-88.
QUERUBIN, L.J., PAGAPAS, V., CENTENO, J.R. \& ILAO, P.I. 1993. Azolla as biological scavenger of geothermal ions II. Boron and arsenic uptake under greenhouse conditions. Philippine Agriculturist 76:89-101.

SANTOS, J.E. \& LACAVA, P.M. 1981. Caracterização e estimativa da atividade da nitrogenase in vitro de bactérias aeróbias fixadoras de nitrogênio. Ciência e Cultura 33:835-838.

SELA, M., FRITZ, E., HUNTERMANN,A. \& TEL-OR, E. 1990. Studies on cadmium localization in the water fern Azolla. Physiologia Plantarum 79:547-553.

SINGH, D.P. \& SINGH, P.K. 1989. The response of rice to the growth and nitrogen fixation in Azolla caroliniana and Azolla pinnata at varying rates of phosphate fertilization. Plant and Soil 114:205-209.

SINGH, D.P. \& SINGH, P.K. 1995. Response of Azolla caroliniana and rice to phosphorus enrichment of Azolla inoculum and phosphorus fertilization during intercropping. Experimental Agriculture 31:21-26.

UHEDA, E. \& NAKAMURA, S. 2000. Abscission of Azolla branches induced by ethylene and sodium azide. Plant Cell Physiology 41:1365-1372. 